\title{
Management of pregnancy, delivery and postpartem care of mothers with ventriculoperitoneal-shunted hydrocephalus and review of literature
}

\author{
B Haeussler*, E Laimer, J Hager, R Haeussler, Ch Keller and Ch Brezinka
}

Address: Univ. Hospital Innsbruck, Anichstr. 35, 6020 Innsbruck

Email: B Haeussler* - beatrice.haeussler@uklibk.ac.at

* Corresponding author

from 49th Annual Meeting of the Society for Research into Hydrocephalus and Spina Bifida

Barcelona, Spain, 29 June - 2 July 2005

Published: 30 December 2005

Cerebrospinal Fluid Research 2005, 2(Suppl I):SI7 doi:I0.II86/I743-8454-2-SI-SI7

\section{Background}

Since the introduction of cerebro spinal fluid (CSF) -shunt implantation in 1971 at the University Hospital of Innsbruck the number of hydrocephalus patients reaching reproductive age has increased in recent years. Our main aim was to attend these patients throughout pregnancy, delivery and maternity. These patients have been in our care from the beginning of their disease to adulthood and we have reviewed their outcome with other reported patients in literature.

\section{Materials and methods}

Follow-up data were obtained either from our own clinical notes or from charts of the obstetrics unit and anaesthesiology unit.

\section{Results}

We present three patients with four pregnancies out of our cohort of 365 CSF- shunted patients. Indications for shunt placement were twice postmeningitis hydrocephalus occlusus (at the age of three months and at the age of two months after premature birth of 34 gestational weeks) and once congenital hydrocephalus of undetermined origin. There were no shunt malfunctions during all four pregnancies. Pregnancy outcomes were three vaginal deliveries, one using vacuum extraction. One woman refused vaginal delivery for her second child and had a primary low transverse cesarean in spinal anaesthesia with likewise no shunt malfunction.

We built up a multidisciplinary team with clearly defined roles, including an obstetrician, a neurologist and an anaesthetist. Because of different correlated mentally impairment of these patients intensive follow-up and special aspects after delivery have to be addressed in order to ensure the quality of care like medical consultation and educational matters concerning the baby, social welfare work, vocational guidance and vocational training to improve their independence.

\section{Conclusion}

Based on our few cases we could not experience complications regarding maternal shunt dependency contrary to literature. All these patients being now "mother patients" are well integrated in society and their families. We suggest that proper care of these patients can lead to normal pregnancies, deliveries and maternity. 